\title{
Duals and Invariances of Frame Sequences
}

\author{
Shannon Bishop ${ }^{a}$, Christopher Heil ${ }^{b}$, Yoo Young Koo ${ }^{c}$, and Jae Kun $\operatorname{Lim}^{d}$ \\ ${ }^{a}$ School of Mathematics, Georgia Institute of Technology, Atlanta, GA 30332, USA; \\ ${ }^{b}$ School of Mathematics, Georgia Institute of Technology, Atlanta, GA 30332, USA; \\ ${ }^{c}$ Department of Mathematics, Yonsei University, Seoul 120-749, Republic of Korea; \\ ${ }^{d}$ Department of Applied Mathematics, Hankyong National University, 67 Seokjeong-dong \\ Anseong-si, Gyeonggi-do, 456-749, Republic of Korea
}

\begin{abstract}
This paper surveys recent results on frame sequences. The first group of results characterizes the relationships that hold among various types of dual frame sequences. The second group of results characterizes the relationships that hold among the major Paley-Wiener perturbation theorems for frame sequences, and some of the properties that remain invariant under such perturbations.
\end{abstract}

Keywords: Bessel sequences, excess, frames, Gabor systems, oblique duals, Type I duals, Type II duals, Riesz bases, shift-invariant spaces, wavelets

\section{INTRODUCTION}

A countable sequence $F=\left\{f_{i}\right\}_{i \in I}$ of elements of a Hilbert space $H$ is a frame for $H$ if there exist constants $A$, $B>0$ such that

$$
\forall f \in H, \quad A\|f\|^{2} \leq \sum_{i \in I}\left|\left\langle f, f_{i}\right\rangle\right|^{2} \leq B\|f\|^{2} .
$$

The numbers $A, B$ are called lower and upper frame bounds, respectively. Frames were first introduced by Duffin and Schaeffer ${ }^{20}$ in the context of nonharmonic Fourier series, and today frames play important roles in many applications in mathematics, science, and engineering. We refer to the texts of Daubechies, ${ }^{19}$ Christensen, ${ }^{13}$ or the research-tutorial of Heil-Walnut ${ }^{24}$ for basic properties of frames.

Each frame $F$ provides basis-like representations of the elements of $H$. Specifically, there exist vectors $\widetilde{f}_{i} \in H$ such that

$$
\forall f \in H, \quad f=\sum_{i \in I}\left\langle f, f_{i}\right\rangle \widetilde{f}_{i}=\sum_{i \in I}\left\langle f, \widetilde{f}_{i}\right\rangle f_{i},
$$

with unconditional convergence of these series. In general, however, a frame need not be a basis, and the representations in (2) need not be unique. Frames which are not bases are overcomplete, i.e., there exist proper subsets of the frame which are complete. ${ }^{20}$ The excess ${ }^{3}$ of the frame is the greatest integer $n$ such that $n$ elements can be deleted from the frame and still leave a complete set, or $\infty$ if there is no upper bound to the number of elements that can be removed. In the former case, it can be shown that the frame is simply a Riesz basis to which finitely many elements have been adjoined. ${ }^{27}$ Such frames are called "near Riesz bases" and behave in many respects like Riesz bases. A frame with infinite excess need not contain a Riesz basis as a subset. ${ }^{11}$

In this paper we are primarily interested in frame sequences, which are frames for their closed spans within a Hilbert space. For example, frames for shift-invariant subspaces are of this type, and play key roles in many applications. We will survey two categories of results that have recently been obtained by the authors.

The first group of results, due to Heil, Koo, and $\mathrm{Lim},{ }^{25}$ characterizes the various kinds of dual sequences that a frame sequence can possess. Christensen and Eldar ${ }^{14}$ and Hemmat and Gabardo ${ }^{26}$ each defined and studied several types of duals (see also Gabardo and $\operatorname{Han}^{21}$ ). We have characterized the exact relationships that hold

E-mail: (S. Bishop) sbishop@math.gatech.edu; (C. Heil) heil@math.gatech.edu; (Y. Y. Koo) yykoo@yonsei.ac.kr; (J. K. Lim) jaekun@hknu.ac.kr 
among these duals, and have obtained results on the uniqueness of dual sequences. Proofs of these results have recently appeared. ${ }^{25}$

The second group of results, due to Bishop, Heil, Koo, and Lim, ${ }^{8}$ determines the exact relationships that hold among the major Paley-Wiener perturbation theorems for frame sequences, and addresses the invariance properties of frame sequences under such perturbations. Major properties of a frame sequence such as excess, deficit, and rank are shown to remain invariant under Paley-Wiener perturbations, but need not be preserved by compact perturbations. For localized frames, which are frames with additional structure, we have shown that the frame measure function is additionally preserved by Paley-Wiener perturbations. The statements of some of these results are announced here, with full details and proofs in a separate research article. ${ }^{8}$

\section{PRELIMINARIES}

$\mathbb{N}$ will denote the set of natural numbers, while $I$ will denote a generic countable index set. $|E|$ denotes the cardinality of a set $E$. $H$ will always denote a separable, infinite-dimensional Hilbert space. We use a Fourier transform normalized as $\widehat{f}(\xi):=\int f(t) e^{-2 \pi i \xi \cdot t} d t$ for $f \in L^{1}\left(\mathbb{R}^{d}\right)$. This extends in the usual way to a unitary operator on $L^{2}\left(\mathbb{R}^{d}\right)$.

The finite linear span of a sequence of elements $F=\left\{f_{i}\right\}_{i \in I}$ of $H$ will be denoted by $\operatorname{span}(F)$. The closure in $H$ of this set will be denoted by $\overline{\operatorname{span}}(F)$. We say that $F$ is complete if $\overline{\operatorname{span}}(F)=H$, or, equivalently, if the only vector $f$ satisfying $\left\langle f, f_{i}\right\rangle=0$ for all $i$ is $f=0$.

A sequence $F=\left\{f_{i}\right\}_{i \in I}$ in $H$ is a Bessel sequence if there exists a constant $B>0$ such that

$$
\forall f \in H, \quad \sum_{i \in I}\left|\left\langle f, f_{i}\right\rangle\right|^{2} \leq B\|f\|^{2} .
$$

In this case the associated analysis operator $T_{F}^{*}: H \rightarrow \ell^{2}(I)$ is defined by $T_{F}^{*}(f)=\left\{\left\langle f, f_{i}\right\rangle\right\}_{i \in I}$, and the synthesis operator or pre-frame operator $T_{F}: \ell^{2}(I) \rightarrow H$ is $T_{F}(c)=\sum c_{i} f_{i}$. This series converges unconditionally in the norm of $H$ for any $c=\left(c_{i}\right)_{i \in I} \in \ell^{2}(I)$. These are everywhere-defined, bounded operators, each adjoint to the other. The elements of a Bessel sequence are uniformly bounded above in norm, with $\left\|f_{i}\right\|^{2} \leq B$ for each $i \in I$.

Comparing equations (1) and (3), we see that every frame is a Bessel sequence. However, a frame possesses additional useful properties. The frame operator $S_{F}=T_{F} T_{F}^{*}: H \rightarrow H$, given by $S_{F} f=\sum\left\langle f, f_{i}\right\rangle f_{i}$, is a positive, continuous, invertible mapping of $H$ onto itself, and satisfies the operator inequalities $A I \leq S_{F} \leq B I$. The canonical dual frame is $\widetilde{F}=\left\{\widetilde{f}_{i}\right\}_{i \in I}$ where $\widetilde{f}_{i}=S_{F}^{-1} f_{i}$. If $F$ has frame bounds $A$, $B$, then the canonical dual frame is a frame with frame bounds $B^{-1}, A^{-1}$. Furthermore, the frame expansions in (2) hold.

We say that a frame $F$ is tight if it is possible to take $A=B$ in (1). It is Parseval if we can take $A=B=1$. The frame operator for a tight frame is $S_{F}=A I$. In particular, if $F$ is a Parseval frame, then $\widetilde{F}=F$.

A Riesz sequence is a sequence $F=\left\{f_{i}\right\}_{i \in I}$ for which there exist $A, B>0$ such that

$$
\forall c \in \ell^{2}(I), \quad A \sum_{i \in I}\left|c_{i}\right|^{2} \leq\left\|\sum_{i \in I} c_{i} f_{i}\right\|^{2} \leq B \sum_{i \in I}\left|c_{i}\right|^{2} .
$$

A Riesz sequence is a frame for its closed span in $H$. A complete Riesz sequence is called a Riesz basis for $H$. If $F$ is a frame, then the frame expansion given in (2) is unique for each $f \in H$ if and only if $F$ is a Riesz basis.

The excess of a sequence $F=\left\{f_{i}\right\}_{i \in I}$ in $H$ is

$$
\operatorname{excess}(F)=\sup \{|G|: G \subseteq F \text { and } \overline{\operatorname{span}}(F \backslash G)=\overline{\operatorname{span}}(F)\} .
$$

The deficit of $F$ is

$$
\operatorname{deficit}(F)=\inf \{|G|: G \subseteq H, \overline{\operatorname{span}}(F \cup G)=H\}
$$




\section{DUALS OF FRAME SEQUENCES}

We will mostly be interested in frame sequences, which are frames for their closed span within $H$. Considering a frame sequence $F=\left\{f_{i}\right\}_{i \in I}$ as a frame for $\operatorname{span}(\mathcal{F})$, it has a canonical dual frame $\widetilde{F}=\left\{\widetilde{f}_{i}\right\}_{i \in I}$ that is a frame for $\overline{\operatorname{span}}(F)$. We call this the canonical dual frame sequence for $F$. We can realize this sequence in terms of the pseudoinverse of the frame operator $S_{F}=T_{F} T_{F}^{*}$. This is a positive operator that is a bounded bijection of $\operatorname{span}(F)$ onto itself. Letting $\dagger$ denotes the pseudoinverse of a bounded operator with closed range, we have $\widetilde{F}=S_{F}^{\dagger}(F)$. Restricted to the domain $H_{F}$, which $S_{F}$ maps invertibly into itself, we have $S_{F}^{\dagger}=S_{F}^{-1}$. The canonical dual is itself a frame sequence, and $H_{\widetilde{F}}=\overline{\operatorname{span}}(\widetilde{F})=\overline{\operatorname{span}}(F)=H_{F}$. Further, we have the reproducing properties

$$
\left.T_{F} T_{\widetilde{F}}^{*}\right|_{H_{F}}=\left.T_{\widetilde{F}} T_{F}^{*}\right|_{H_{F}}=\left.I\right|_{H_{F}}
$$

where $I$ is the identity operator on $H$. Equation (4) is equivalent to

$$
f=\sum_{i \in I}\left\langle f, \widetilde{f}_{i}\right\rangle f_{i}=\sum_{i \in I}\left\langle f, f_{i}\right\rangle \widetilde{f}_{i}, \quad f \in H_{F},
$$

and furthermore these series converge unconditionally.

The canonical dual frame sequence is usually not the only "dual sequence." Any sequence $G=\left\{g_{i}\right\}_{i \in I}$ in $H$ that has the property that

$$
f=T_{F} T_{G}^{*} f=\sum_{i \in I}\left\langle f, g_{i}\right\rangle f_{i}, \quad f \in H_{F},
$$

is called a dual sequence to $F$. Out of all the possible dual sequences, some have special properties. In particular, Type I and Type II duals were introduced by Hemmat and Gabardo, ${ }^{26}$ while Christensen and Eldar ${ }^{14}$ introduced oblique duals. These are defined precisely as follows.

Definition 3.1. Let $F=\left\{f_{i}\right\}_{i \in I}$ be a frame sequence and $G=\left\{g_{i}\right\}_{i \in I}$ a Bessel sequence in $H$.

(a) $G$ is a generalized or alternate dual of $F$, or simply a dual for short, if

$$
\left.T_{F} T_{G}^{*}\right|_{H_{F}}=\left.I\right|_{H_{F}} .
$$

(b) $G$ is a Type I dual of $F$ if $G$ is a dual and range $\left(T_{G}\right) \subseteq \operatorname{range}\left(T_{F}\right)$.

(c) $G$ is a Type II dual of $F$ if $G$ is a dual and $\operatorname{range}\left(T_{G}^{*}\right) \subseteq \operatorname{range}\left(T_{F}^{*}\right)$.

(d) $G$ is an oblique dual of $F$ if $G$ is a frame sequence that is dual to $F$, and also $F$ is dual to $G$.

Note that while $F$ and $G$ play symmetric roles in the definition of oblique dual, the definition of Type I and Type II duals does not seem to be symmetric in $F$ and $G$. The next theorem demonstrates that the Type I and Type II definitions are indeed symmetric, and in fact are special cases of oblique duals. We write $\oplus$ to denote an orthogonal direct sum of closed subspaces, and $\dot{+}$ to denote a direct sum of closed subspaces whose intersection is zero. Part (a) of this theorem was known previously. ${ }^{13}$ Part (b) was first proved by the authors. ${ }^{25}$

TheOREm 3.2. Let $F$ be a frame sequence and $G$ a Bessel sequence in $H$.

(a) If $G$ is a Type I dual of $F$, then $G$ is a frame sequence and $F$ is a Type I dual of $G$. In this case, $F$ and $G$ are oblique duals, $H_{G}=\operatorname{range}\left(T_{G}\right)=\operatorname{range}\left(T_{F}\right)=H_{F}$, and $H=\operatorname{range}\left(T_{F}\right) \oplus \operatorname{range}\left(T_{G}\right)^{\perp}$.

(b) If $G$ is a Type II dual of $F$, then $G$ is a frame sequence and $F$ is a Type II dual of $G$. In this case, $F$ and $G$ are oblique duals, $\operatorname{range}\left(T_{G}^{*}\right)=\operatorname{range}\left(T_{F}^{*}\right)$, and $H=\operatorname{range}\left(T_{F}\right) \dot{+} \operatorname{range}\left(T_{G}\right)^{\perp}$. 
Thus Theorem 3.2 clarifies the interest of these types of duals. An oblique dual $G$ is one where $F$ and $G$ play completely complementary roles, with each being a frame sequence that is dual to the other. Type I and Type II duals are oblique duals with further restrictions. In particular, if it is important that $F$ and $G$ have the same closed spans, then we should look for a Type I dual. On the other hand, the flexibility offered by frame expansions can often be better utilized by looking for a dual that is not within $H_{F}$. This is important in many applications. ${ }^{16,29}$ A Type II dual will instead require that $\operatorname{ker}\left(T_{F}\right)$ and $\operatorname{ker}\left(T_{G}\right)$ be equal, rather than the ranges of $T_{F}$ and $T_{G}$. The canonical dual is simultaneously a Type I and a Type II dual.

The relations that hold between the various types of duals are explained in the next result. ${ }^{25}$

THEOREM 3.3. There are examples of a frame sequence $F$ with dual $G$ such that:

(a) $G$ is not a frame sequence;

(b) $G$ is a frame sequence but not a Type I dual;

(c) $G$ is a frame sequence but not a Type II dual;

(d) G is a Type I dual but not a Type II dual of F;

(e) G is a Type II dual but not a Type I dual of F;

(f) $G$ is an oblique dual but not a Type I dual of F;

(g) $G$ is an oblique dual but not a Type II dual of F.

The following theorem characterizes the existence of oblique or Type II duals in terms of direct sum decompositions of $H .^{25}$

TheOrem 3.4. Let $U, V$ be closed subspaces of $H$, and let $F$ be a frame for $U$. Then the following statements are equivalent.

(a) $H=U \dot{+} V^{\perp}$.

(b) There is a frame $G$ for $V$ that is a Type II dual of $F$.

(c) There is a frame $G$ for $V$ that is an oblique dual of $F$.

In case these hold, $P F$ is a frame for $V$ and we can take $G$ to be the canonical dual frame of PF in $V$, where $P=\left.P_{V}\right|_{U}: U \rightarrow V$ is the restriction of the orthogonal projection $P_{V}$ onto $V$ to the domain $U$.

\section{INVARIANCES OF FRAME SEQUENCES}

Now we turn to recent results on perturbation of frame sequences. These will be phrased in terms of the inf-angle $R(U, V)$ between closed subspaces $U, V$ of $H$. When $U$ is nontrivial, this is defined ${ }^{31}$ by

$$
R(U, V)=\inf _{u \in U \backslash\{0\}} \frac{\left\|P_{V} u\right\|}{\|u\|},
$$

For $U=\{0\}$ we define $R(\{0\}, V)=1$.

The following theorem relates the inf-angle to projections. The equivalence of parts (a), (e), and (f) is stated by Tang. ${ }^{30}$ The equivalence of these parts with (b), (c), and (d) is proved by the authors. ${ }^{8}$ We write $U \cong V$ to mean that $U$ is isomorphic to $V$, which is the case if and only if $U$ and $V$ have the same dimension. In the statement of this theorem the symbols $\left.P_{U}\right|_{V}$ denote the restriction of the orthogonal projection $P_{U}$ to the domain $V$.

TheOREM 4.1. Let $U, V$ be closed subspaces of $H$ with at least one nontrivial. Then the following statements are equivalent. 
(a) $0<R(U, V)$ and $0<R(V, U)$.

(b) $0<R(U, V)=R(V, U)$.

(c) $\left.P_{V}\right|_{U}: U \rightarrow V$ is invertible.

(d) $\left.P_{U}\right|_{V}: V \rightarrow U$ is invertible.

(e) $H=U \dot{+} V^{\perp}$.

(f) $H=V \dot{+} U^{\perp}$.

Moreover, in case these hold, we have $U \cong V, V^{\perp} \cong U^{\perp}$, and

$$
0<R(U, V)=R(V, U)=\left\|\left(\left.P_{V}\right|_{U}\right)^{-1}\right\|^{-1}=\left\|\left(\left.P_{U}\right|_{V}\right)^{-1}\right\|^{-1} .
$$

The following result summarizes the main Paley-Wiener-type perturbation theorems known to hold for frame sequences. These are due to Christensen and his co-authors. ${ }^{12,15,17}$

THEOREM 4.2. Let $F=\left\{f_{i}\right\}_{i \in I} \subseteq H$ be a frame sequence in $H$, with synthesis operator $T_{F}$ and frame bounds $A_{F}, B_{F}$. Let $G=\left\{g_{i}\right\}_{i \in I} \subseteq H$ be another sequence with synthesis operator $T_{G}$, and let $\mu \geq 0$ be fixed. Define $H_{F}=\overline{\operatorname{span}}(F)=\operatorname{range}\left(T_{F}\right)$ and $H_{G}=\overline{\operatorname{span}}(G)$. If

$$
\left\|T_{F} c-T_{G} c\right\| \leq \mu\|c\| \text { for all finitely supported } c \in \ell^{2}(I),
$$

then $G$ is a Bessel sequence. Moreover, if any one of the following conditions on the inf-angle between subspaces holds, then $G$ is a frame sequence:

(i) $\mu<\sqrt{A_{F}} R\left(\operatorname{ker}\left(T_{F}\right), \operatorname{ker}\left(T_{G}\right)\right)$,

(ii) $\mu<\sqrt{A_{F}} R\left(H_{G}, H_{F}\right)$,

(iii) $\mu<\sqrt{A_{F}}$ and $R\left(H_{G}, H_{F}\right)>0$.

In fact, there are more complicated theorems involving not only $\mu$ but also two more non-negative constants. ${ }^{10,12,17,28}$ However, the remaining parameters are rarely (if ever) used in applications of the perturbation theorems to wavelet, Gabor, shift-invariant, or exponential frames. ${ }^{1,18}$ Therefore, we focus on the above "practical" versions of the perturbation theorems.

The next theorem characterizes the exact implications that hold among the hypothesis (i), (ii), and (iii) in the Paley-Wiener-type perturbation theorems. ${ }^{8}$ Moreover, these types of perturbations preserve many of the fundamental properties of frames, including rank, excess, and deficit. Thus, Paley-Wiener-type perturbations preserve the "size" of a frame sequence in many ways.

THEOREM 4.3. Let a frame sequence $F$ and a sequence $G$ be given as in Theorem 4.2, and suppose that equation (7) is satisfied. Then the following statements hold.

- (i) implies (iii), but not vice versa.

- (ii) implies (iii), but not vice versa.

- (i) and (ii) are independent. 
Moreover, if any one of (i), (ii), or (iii) is satisfied, then $G$ is a frame sequence, and the following statements hold.

- $H_{F}$ is isomorphic to $H_{G}$.

- $H_{F}^{\perp}$ is isomorphic to $H_{G}^{\perp}$.

- $\operatorname{ker}\left(T_{F}\right)$ is isomorphic to $\operatorname{ker}\left(T_{G}\right)$.

- $\operatorname{rank}(F)=\operatorname{rank}(G)$.

- $\operatorname{excess}(F)=\operatorname{excess}(G)$.

- $\operatorname{deficit}(F)=\operatorname{deficit}(G)$.

Some of the implications in Theorem 4.3 were known previously. The important new points are the implication (i) implies (iii) and the invariance conclusions that follow when the weakest condition (iii) holds. The fact that $H_{F}$ is isomorphic to $H_{G}$ and $H_{F}^{\perp}$ is isomorphic to $H_{G}^{\perp}$ if (ii) is satisfied is proved by Christensen, Lennard, and Lewis, ${ }^{17}$ and the fact that $H_{F}$ is isomorphic to $H_{G}$ if (iii) is satisfied is proved by Christensen, Kim, Kim, and Lim. ${ }^{15}$ The implication (ii) implies (iii) is trivial because of the fact that $R\left(H_{G}, H_{F}\right) \leq 1$. The paper by Koo and $\mathrm{Lim}^{28}$ also contains some of the implications of Theorem 4.3 for the restricted setting of shift-invariant frame systems. Additionally, the counterexamples constructed there establish the "not vice versa" statements in Theorem 4.3 as well as the independence of (i) and (ii).

Excess, deficit, and rank are measures of the overcompleteness or undercompleteness of a frame sequence, but only in a relatively crude sense. For arbitrary frames, it is extremely difficult to quantify the exact meaning of redundancy. However, many practical frames have a structure which allow more precise statements to be made. For example, a Gabor frame sequence is a frame sequence of the form

$$
\mathcal{G}(g, \Lambda)=\left\{e^{2 \pi i \beta x} g(x-\alpha)\right\}_{(\alpha, \beta) \in \Lambda}
$$

where $g \in L^{2}(\mathbb{R})$ and $\Lambda \subseteq \mathbb{R}^{2}$ are fixed. A longstanding folklore for Gabor frames is that the density of the index set $\Lambda$ equals the redundancy of the frame $\mathcal{G}(g, \Lambda)$. Density of the indexed set is defined in terms of Beurling density, which is in a sense the average number of points of $\Lambda$ that lie in a unit cube (e.g., for the lattice $\Lambda=\alpha \mathbb{Z} \times \beta \mathbb{Z}$ the Beurling density is $1 /(\alpha \beta)$ ). For a survey of the long history of results connected to Beurling density and the Nyquist Theorem for Gabor frames, including precise definitions and an extensive bibliography, we refer to the paper by Heil. ${ }^{23}$

Recently, the introduction of the concept of localized frames has allowed this folklore to be given a quantitative interpretation. Localized frames were introduced independently by Gröchenig ${ }^{22}$ and by Balan, Casazza, Heil, and Landau, ${ }^{4}$ for quite different purposes. Applied to Gabor frames, ${ }^{4,5}$ these results imply, for example, that any localized frame (including Gabor frames) must have lower density of at least 1, and if such a frame is a union of $N$ Riesz bases then the upper and lower densities of $\Lambda$ are exactly $N$. A deep new result by Balan, Casazza, and Landau ${ }^{6}$ is that if the density of a localized frame is $d>1$ then there exists a subset of the frame with density $1+\varepsilon$ that is still a frame for the space. These results and others show that the density $d$ of a localized frame, which is determined solely by the index set alone, quantifies the redundancy of the frame.

Closely related to density and redundancy issues is the question of when frame sequences are equivalent. A naive notion of equivalence for frame sequences is that $F=\left\{f_{i}\right\}_{i \in I}$ and $G=\left\{g_{i}\right\}_{i \in I}$ are equivalent ${ }^{2}$ if there exists a bounded bijection $U: H \rightarrow H$ such that $U\left(f_{i}\right)=g_{i}$ for each $i$. This is the correct notion of equivalence for bases, but because of the redundancies inherent in frames, this notion of equivalent frames is too strong. For example, frames that are identical except for the ordering of their index set need not be equivalent under this definition, even though the definition and most properties of frames are independent of ordering. A new notion of frame equivalence based on the idea of a frame measure function was introduced by Balan and Landau. ${ }^{7}$ Two frames are equivalent in this definition if their frame measure functions coincide. This notion of equivalence is 
independent of the ordering of the index set, under multiplication of frame elements by scalars of unit modulus, and other seemingly trivial modifications that were not invariant under the earlier notion of frame equivalence.

The next theorem concerns the behavior of the frame measure function under Paley-Wiener-type perturbations. In this theorem, $P_{F}$ denotes the orthogonal projection of $H$ onto $H_{F}$, and $\left.P_{F}\right|_{G}$ denotes the restriction of $P_{F}$ to $H_{G}$. For the precise definition of localization and the frame measure function, we refer to the abovementioned articles. ${ }^{7,8}$

THEOREM 4.4. Let $F=\left\{f_{n}\right\}_{n \in \mathbb{Z}}$ be a frame sequence in $H$ with frame bounds $A_{F}, B_{F}$, and let $G=\left\{g_{n}\right\}_{n \in \mathbb{Z}}$ be a sequence in H. Suppose that equation (7) and hypothesis (iii) from Theorem 4.2 are satisfied, so $G$ is a frame sequence. Let $\left\{\widetilde{g}_{n}\right\}_{n \in \mathbb{Z}}$ be the canonical dual frame sequence for $G$ within $H_{G}$. Then, in addition to the conclusions of Theorem 4.3, the following statements hold.

(a) $P_{F} G=\left\{P_{F} g_{n}\right\}_{n \in \mathbb{Z}}$ is a frame for $H_{F}$, and its canonical dual frame sequence is $\left\{\left(\left.P_{G}\right|_{F}\right)^{-1} \widetilde{g}_{n}\right\}_{n \in \mathbb{Z}}$.

(b) If $(F, G)$ is $\ell^{p}$-localized, then $\left(F, P_{F} G\right)$ is $\ell^{p}$-localized.

(c) If $\left(F, P_{F} G\right)$ is $\ell^{2}$-localized then $F, P_{F} G$, and $G$ all have the same frame measure function and hence are equivalent in the sense of. ${ }^{7}$

\section{ACKNOWLEDGMENTS}

The first and second authors were partially supported by NSF Grant DMS-0806532. The third author was supported by Korea Research Foundation Grant KRF-2007-357-C00005, funded by the Korean Government (MOEHRD). The fourth author was supported by a research grant from Academic Research Foundation of Hankyong National University for a scholarly exchange program in 2007.

\section{REFERENCES}

[1] R. Balan, "Stability theorems for Fourier frames and wavelet Riesz bases," J. Fourier Anal. Appl., 3, 499-504 (1997).

[2] R. Balan, "Equivalence relations and distances between Hilbert frames," Proc. Amer. Math. Soc., 127, 2353-2366 (1999).

[3] R. Balan, P. G. Casazza, C. Heil, and Z. Landau, "Deficits and excesses of frames," Adv. Comput. Math., 18, 93-116 (2003).

[4] R. Balan, P. G. Casazza, C. Heil, and Z. Landau, "Density, overcompleteness, and localization of frames, I. Theory," J. Fourier Anal. Appl., 12, 105-143 (2006).

[5] R. Balan, P. G. Casazza, C. Heil, and Z. Landau, Density, overcompleteness, and localization of frames, II. Gabor frames, J. Fourier Anal. Appl., 12, 307-344 (2006).

[6] R. Balan, P. G. Casazza, and Z. Landau, "Redundancy for localized frames," preprint (2009).

[7] R. Balan and Z. Landau, "Measure functions for frames," J. Funct. Anal., 252, 630-676 (2007).

[8] S. Bishop, C. Heil, Y. Y. Koo, and J. K. Lim, "Invariances of frame sequences under perturbtations," preprint (2009).

[9] P. G. Casazza, "Modern tools for Weyl-Heisenberg (Gabor) frame theory," Adv. Imag. Electron Phy., 115, 1-127 (2000).

[10] P. G. Casazza and O. Christensen, "Perturbation of operators and applications to frame theory," J. Fourier Anal. Appl., 5, 543-557 (1997).

[11] P. G. Casazza and O. Christensen, "Frames containing a Riesz basis and preservation of this property under perturbations," SIAM J. Math. Anal., 29, 266-278 (1998).

[12] O. Christensen, "Operators with closed range, pseudo-inverses, and perturbation of frames for a subspace," Canad. Math. Bull., 42, 37-45 (1999).

[13] O. Christensen, An Introduction to Frames and Riesz Bases, Birkhäuser, Boston, 2003. 
[14] O. Christensen and Y. C. Eldar, "Oblique dual frames and shift-invariant spaces," Appl. Comput. Harmon. Anal., 17, 48-68 (2004).

[15] O. Christensen, H. O. Kim, R. Y. Kim, and J. K. Lim, "Perturbation of frame sequences in shift-invariant spaces," J. Geom. Anal., 15, 181-191 (2005).

[16] O. Christensen and R. Y. Kim, "Pairs of explicitly given dual Gabor frames in $L^{2}\left(\mathbb{R}^{d}\right)$," J. Fourier Anal. Appl., 12, 243-255 (2006).

[17] O. Christensen, C. Lennard, and C. Lewis, "Perturbation of frames for a subspace of a Hilbert space," Rocky Mountain J. Math., 30, 1237-1249 (2000).

[18] O. Christensen and W. Sun, "Stability of wavelet frames with matrix dilations," Proc. Amer. Math. Soc., 134, 831-842 (2006).

[19] I. Daubechies, Ten Lectures on Wavelets, SIAM, Philadelphia, PA, 1992.

[20] R. J. Duffin and A. C. Schaeffer, "A class of nonharmonic Fourier series," Trans. Amer. Math. Soc., 72, 341-366 (1952).

[21] J.-P. Gabardo and D. Han, "The uniqueness of the dual of Weyl-Heisenberg subspace frames," Appl. Comput. Harmon. Anal., 17, 226-240 (2004).

[22] K. Gröchenig, "Localization of frames, Banach frames, and the invertibility of the frame operator," J. Fourier Anal. Appl., 10, 105-132 (2004).

[23] C. Heil, "History and evolution of the Density Theorem for Gabor frames," J. Fourier Anal. Appl., 13, 113-166 (2007).

[24] C. E. Heil and D. F. Walnut, "Continuous and discrete wavelet transforms," SIAM Rev., 31, 628-666 (1989).

[25] C. Heil, Y. Y. Koo, and J. K. Lim, "Duals of frame sequences," Acta Appl. Math., 107, 75-90 (2009).

[26] A. A. Hemmat and J.-P. Gabardo, "The uniqueness of shift-generated duals for frames in shift-invariant subspaces," J. Fourier Anal. Appl., 13, 589-606 (2007).

[27] J. R. Holub, "Pre-frame operators, Besselian frames, and near-Riesz bases in Hilbert spaces," Proc. Amer. Math. Soc., 122, 779-785 (1994).

[28] Y. Y. Koo and J. K. Lim, "Perturbation of frame sequences and its applications to shift-invariant spaces," Linear Algebra Appl., 420, 295-309 (2007).

[29] S. Li and H. Ogawa, "Pseudoframes for subspaces with applications," J. Fourier Anal. Appl., 10, 409-431 (2004).

[30] W.-S. Tang, "Oblique projections, biorthogonal Riesz bases and multiwavelets in Hilbert spaces," Proc. Amer. Math. Soc., 128, 463-473 (2000).

[31] M. Unser and A. Aldroubi, "A general sampling theory for non-ideal acquisition devices," IEEE Trans. Signal Process., 42, 2915-2925 (1994). 